\title{
MODELO DE AVALIAÇÃO DE ABORDAGENS EDUCACIONAIS EM REALIDADE AUMENTADA MÓVEL
}

\author{
Fabrício Herpich, PPGIE, UFRGS, fabricio_herpich@hotmail.com \\ Felipe Becker Nunes, AMF, nunesfb@gmail.com \\ Giani Petri, UFSM, gpetri@inf.ufsm.br \\ Priscila Nicolete, PPGIE, UFRGS, priscilanicolete@hotmail.com
}

Liane Margarida Rockenbach Tarouco, PPGIE, UFRGS, liane@penta.ufrgs.br

\begin{abstract}
Resumo. A inserção de tecnologias no âmbito educacional tem fomentado novos recursos e experiências educacionais aos seus usuários, capazes de auxiliar no processo de ensino e aprendizagem em diferentes áreas. Dentre as tecnologias emergentes, o uso de realidade aumentada em dispositivos móveis tem se destacado, em virtude da combinação entre os recursos de multimídia aumentada e de aprendizagem móvel. No entanto, é essencial que essas abordagens educacionais sejam devidamente avaliadas, a fim de obter evidências de sua qualidade. Este artigo tem como objetivo apresentar a proposta de um modelo de avaliação de abordagens educacionais em realidade aumentada móvel, desenvolvido a partir de estudos prévios e de uma metodologia para a definição do objetivo de avaliação, fatores de qualidade, dimensões e itens de avaliação. $O$ modelo foi avaliado por 15 especialistas relacionados à área de concentração do instrumento e validado com base em suas asserções. Os resultados obtidos foram satisfatórios e permitiram validar a construção do modelo para futuras aplicações no âmbito educacional.
\end{abstract}

Palavras-chave: Modelo de Avaliação; Realidade Aumentada; Aprendizagem Móvel; Tecnologia Educacional.

\section{EVALUATION MODEL OF MOBILE AUGMENTED REALITY EDUCATIONAL APPROACHES}

\begin{abstract}
The insertion of technologies in the educational scope has fomented new educational resources and experiences to its users, able to aid in the process of teaching and learning in different areas. Among emerging technologies, the use of augmented reality on mobile devices has stood out because of the combination of enhanced multimedia features and mobile learning. However, it is essential that these educational approaches are properly evaluated in order to obtain evidence of their quality. This paper aims to present the proposal of a model of evaluation of educational approaches in mobile augmented reality, developed from previous studies and a methodology for the definition of the objective of evaluation, quality factors, dimensions, and evaluation items. The model was evaluated by 15 experts related to the area of concentration of the instrument and validated based on its assertions. The results were satisfactory and allowed to validate the construction of the model for future applications in the educational scope.
\end{abstract}

Keywords: Evaluation Model; Augmented Reality; Mobile Learning; Educational Technology. 


\section{INTRODUÇ̃̃̃O}

Nas últimas duas décadas, aplicações de realidade aumentada têm recebido cada vez mais atenção, e seu uso tem atingido diferentes áreas do conhecimento. Esses recursos têm permitido o desenvolvimento de novas interfaces que estão mudando a forma como as organizações apresentam seus conteúdos, aumentando a eficiência e promovendo a inovação (Becker et al, 2017).

A realidade aumentada consiste na sobreposição de informações significativas em um fluxo de vídeo para criar uma experiência visual enriquecida para usuários (Maiti, Maxwell \& Kist, 2017). Chen et al. (2017) explicam que para a educação essa técnica aproveita de objetos virtuais integrados a ambientes físicos, resultando em uma realidade mista na qual objetos virtuais e físicos coexistem de maneira significativa para aumentar as experiências de aprendizagem. O relatório da New Media Consortium - Horizon Report (Becker et al, 2017) destaca a realidade mista como um desenvolvimento importante na área educacional para os próximos anos, por meio de espaços híbridos capazes de proporcionar cenários inovadores, oferecendo aos usuários contato com conteúdos que não seriam possíveis nas salas de aula tradicionais.

O crescimento do uso da realidade aumentada na educação muito se dá pela popularização das tecnologias móveis que estão permitindo o acesso desse tipo de recurso em dispositivos como smartphones e tablets. É possível observar o surgimento da Mobile Augmented Reality (MAR) que alia aspectos da realidade aumenta a aprendizagem móvel (Chatzopoulos et al., 2017). MAR utiliza de recursos contidos em smartphones e tablets, tais como câmera e sensores como GPS, para o reconhecimento e sobreposição dos objetos virtua is no mundo fisico. Para Craig (2017) esse conceito pode representar economia e flexibilidade na educação, uma vez que os estudantes possuem e utilizam estes dispositivos no dia-a-dia.

Atualmente, diversas pesquisas têm adotado realidade aumentada em contextos educacionais. Frank \& Kapila (2017) destacam os recursos de detecção, armazenamento, computação e comunicação dos dispositivos móveis como forma para superar as limitações de equipamentos de laboratório, melhorando a relação custo-beneficio e a portabilidade de plataformas para orientar práticas laboratoriais. Yip et al. (2019) implementaram um ambiente de laboratório para a disciplina de têxtil e vestuário. Os autores desenvolveram um vídeo 360 graus com princípios de realidade aumentada que fornece gráficos e informações sobrepostos no ambiente real. Outros estudos têm utilizado a realidade aumentada para aumentar a sensação de realismo e melhorar o feedback de laboratórios remotos que são utilizados para promover práticas laboratoriais em disciplinas STEM - Science, Technology, Engineering and Mathematics (Maiti, Maxwell \& Kist, 2017; Borrero et al. (2013); Odeh, Shanab \& Anabtawi (2015). Nesses casos, a realidade aumentada é utilizada para adicionar módulos virtuais que podem interagir com hardwares dos laboratórios remotos de forma bidirecional.

No entanto, uma limitação identificada no uso da realidade aumentada aplicada a educação, consiste na avaliação destas abordagens pedagógicas. Ibáñez \& Delgado-Kloos (2018) explicam que em muitos casos, as avaliações são realizadas com questionários desenvolvidos de forma ad-hoc pelos autores, sem uma definição clara do que pretendem medir. Essa limitação também é evidenciada em um estudo prévio realizado pelos autores desta pesquisa no formato de uma revisão sistemática da literatura de abordagens educacionais em realidade aumentada móvel (Herpich et al., 2019), em que foram encontrados diferentes modelos de avaliação de abordagens educacionais em realidade aumentada móvel e inúmeros instrumentos adaptados de outros modelos de avaliação de tecnologias na educação de maneira geral (e.g. jogos educacionais, laboratórios virtuais, entre outros).

Entretanto, a grande maioria desses modelos não contemplam aspectos relacionados à aprendizagem móvel (e.g. conectividade, tamanho de tela e capacidade de processamento) em 


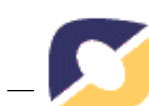

abordagens de realidade aumentada, tampouco abrangem fatores de qualidade que influenciam na aprendizagem dos estudantes (e.g. aspectos pedagógicos, engajamento de usuários, usabilidade de recursos educacionais, aceitação da tecnologia, experiência do usuário, entre outros) em abordagens em realidade aumentada móvel.

Diante disso, o objetivo deste estudo é apresentar o desenvolvimento de um modelo para a avaliação da qualidade de abordagens educacionais em realidade aumentada móvel. Dessa forma, oferecendo um suporte metodológico para que instrutores e pesquisadores possam avaliar abordagens em realidade aumentada móvel utilizadas em contextos educacionais. E, assim, permitindo a identificação sistemática dos pontos fortes e oportunidades de melhorias no uso de tais abordagens no processo de ensino-aprendizagem.

\section{METODOLOGIA}

Os procedimentos metodológicos adotados para a condução desta investigação consistem em diversas etapas, ilustradas na Figura I, que resultaram no Modelo de Avaliação de Abordagens Educacionais em Realidade Aumentada Móvel (Evaluation model of Mobile Augmented Reality Educational Approaches - MAREEA):

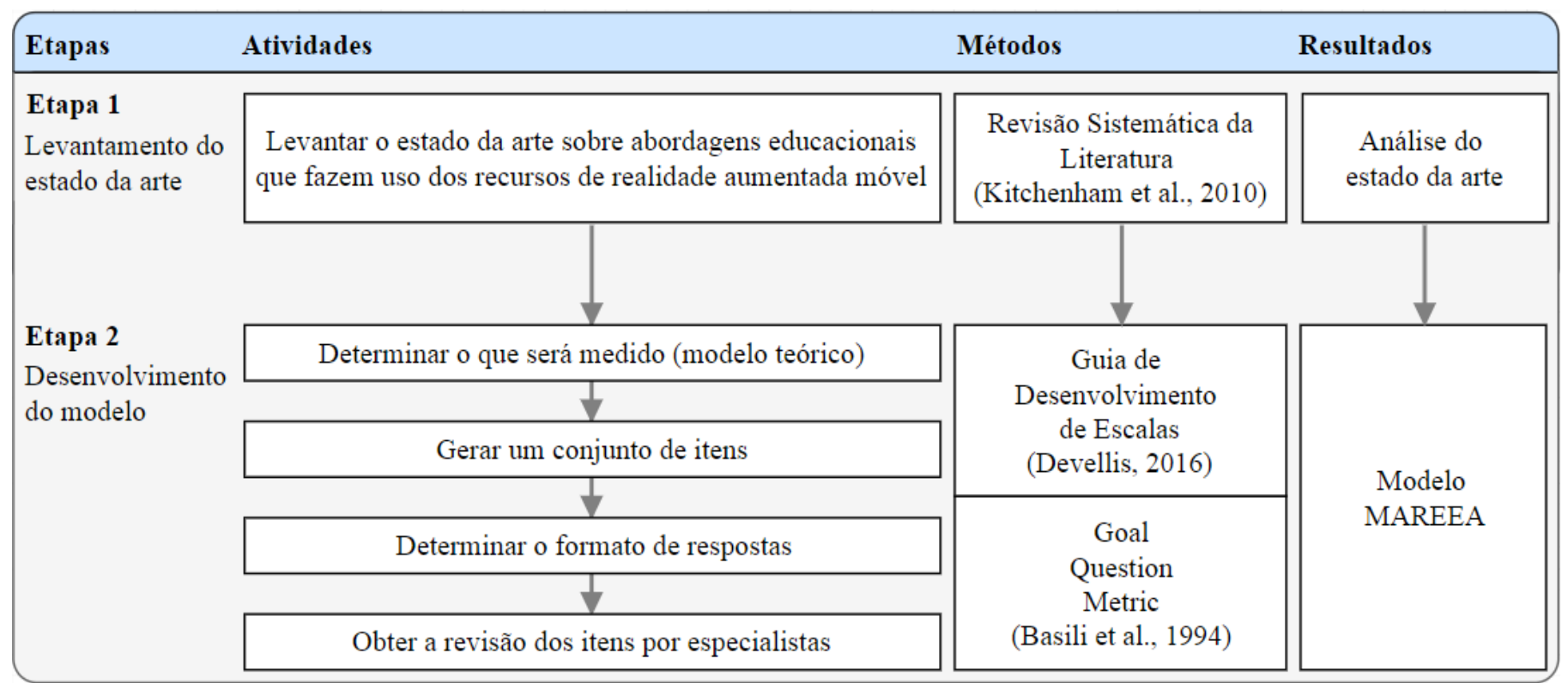

Figura I. Metodologia da Pesquisa

Etapa 1 - Levantamento do Estado da Arte. Inicialmente, foi realizada uma revisão sistemática da literatura com o objetivo de identificar o estado da arte sobre as abordagens educacionais existentes, que combinam os recursos de realidade aumentada com aprendiza gem móvel. Como resultado, identificou-se que a maioria dos modelos de avaliação foram adaptados de outros contextos de avaliação, sem contemplar a avaliação da aprendiza gem móvel e os recursos de realidade aumentada. A descrição detalhada da revisão sistemática da literatura e seus resultados podem ser encontrados em Herpich et al. (2019).

Etapa 2 - Desenvolvimento do Modelo MAREEA. Essa etapa corresponde à construção do modelo MAREEA, tendo como base os resultados do levantamento do estado da arte (Etapa 1). O modelo MAREEA é desenvolvido seguindo uma versão adaptada da metodologia para o desenvolvimento de escalas definido por DeVellis (2016). As etapas adotadas, foram: 
Determinar o que será medido. Com base nos resultados do estado da arte Herpich et al. (2019) e seguindo a abordagem GQM (Goal/Question/Metric) (Basili et al., 1994), são definidos os fatores de avaliação e sistematicamente decompostos em dimensões que apresentam um refinamento dos fatores.

Gerar um conjunto de itens. Cada dimensão definida é sistematicamente decomposta em itens para compor o instrumento de coleta de dados (questionário). E, assim, sistematicamente coletar dados de cada dimensão avaliada.

Determinar o formato das respostas. Como formato de respostas é definido o uso da escala Likert (de 5 pontos) em razão da ampla utilização e aceitação em pesquisas científicas, com uma escala variando de (-2) discordo totalmente, (-1) discordo parcialmente, (0) indiferente, $(+1)$ concordo parcialmente, e, $(+2)$ concordo totalmente. Optou-se por construir uma escala com 5 pontos, com opções negativas e positivas em razão de permitir uma precisão maior nas respostas, fornecendo um ponto neutro na escala, de modo a não afetar a obrigatoriedade de uma escolha.

Obter a revisão dos itens por especialistas. A opinião de especialistas sobre a validade aparente do instrumento de avaliação proposto foi consultada, para que fosse possível identificar adequações necessárias e estabelecer uma padronização da linguagem empregada na redação dos itens. Para este processo de validação, 15 professores doutores e candidatos da área de informática na educação foram convidados a analisarem o instrumento e apresentarem seus pareceres. Demais detalhes referentes à forma de condução e análise dos resultados serão apresentados na Seção 4.

Se torna importante ressaltar que a versão do questionário apresentada neste artigo já considera as sugestões dos especialistas. DeVellis (2016) ainda determina outras três etapas, que consistem em administrar os itens para um grupo de indivíduos, avaliação da validade e confiabilidade dos itens e na otimização do tamanho do instrumento de avaliação, que estão em fase de execução e fora do escopo deste artigo.

\section{MODELO DE AVALIAÇÃO MAREEA}

O modelo MAREEA tem como objetivo analisar as abordagens educacionais em realidade aumentada móvel com o propósito de avaliar a percepção da qualidade em termos de experiência de uso e de aprendizagem dos seus usuários após a interação com tais recursos em contexto educacional.

A partir do objetivo de avaliação foram definidos os fatores de qualidade a serem avaliados nas abordagens educacionais de realidade aumentada móvel. Os fatores de qualidade que compõem o modelo de avaliação MAREEA foram definidos a partir do estado da arte obtido em uma revisão sistemática da literatura Herpich et al. (2019). Com base nos resultados do estado da arte, quatro fatores de avaliação foram definidos para o modelo MAREEA:

Usabilidade: A usabilidade consiste em o quão intuitivo e fácil é para os indivíduos aprenderem a usar e interagir com um produto (Preece, 2001). No contexto de sistemas educacionais, a usabilidade pode tornar os sistemas mais fáceis de usar e permitir que eles se adaptem mais aos requisitos dos usuários.

Engajamento: Jacques, Preece \& Carey (1995) afirmam que o engajamento consiste em uma resposta do usuário para uma interação que mantém ganhos e incentiva a sua atenção, especialmente quando o mesmo se encontra intrinsecamente motivado.

Motivação: A motivação é geralmente definida como aquela que explica a direção e a magnitude do comportamento, ou, em outras palavras, explica quais objetivos as pessoas escolhem para buscar e quão ativamente ou intensamente elas as perseguem (Keller, 1987). 
Aprendizagem Ativa: A aprendizagem ativa é geralmente definida como qualquer método instrucional que envolve os sujeitos no processo de aprendizagem. Em suma, a aprendizagem ativa exige que os indivíduos façam atividades de aprendizagem significativas e pensem sobre o que estão fazendo (Prince, 2004).

Posteriormente, os fatores de qualidade foram decompostos em dimensões de avaliação. A partir da estrutura do modelo MAREEA, as dimensões são sistematicamente decompostas em itens para compor o instrumento de medição (questionário) do modelo MAREEA. Os itens do questionário de avaliação foram definidos com base em questionários padronizados encontrados na literatura que se propõem avaliar de maneira específica cada fator de qualidade, os quais foram adaptados ao contexto de avaliação de abordagens educacionais em realidade aumentada móvel (Figura II).

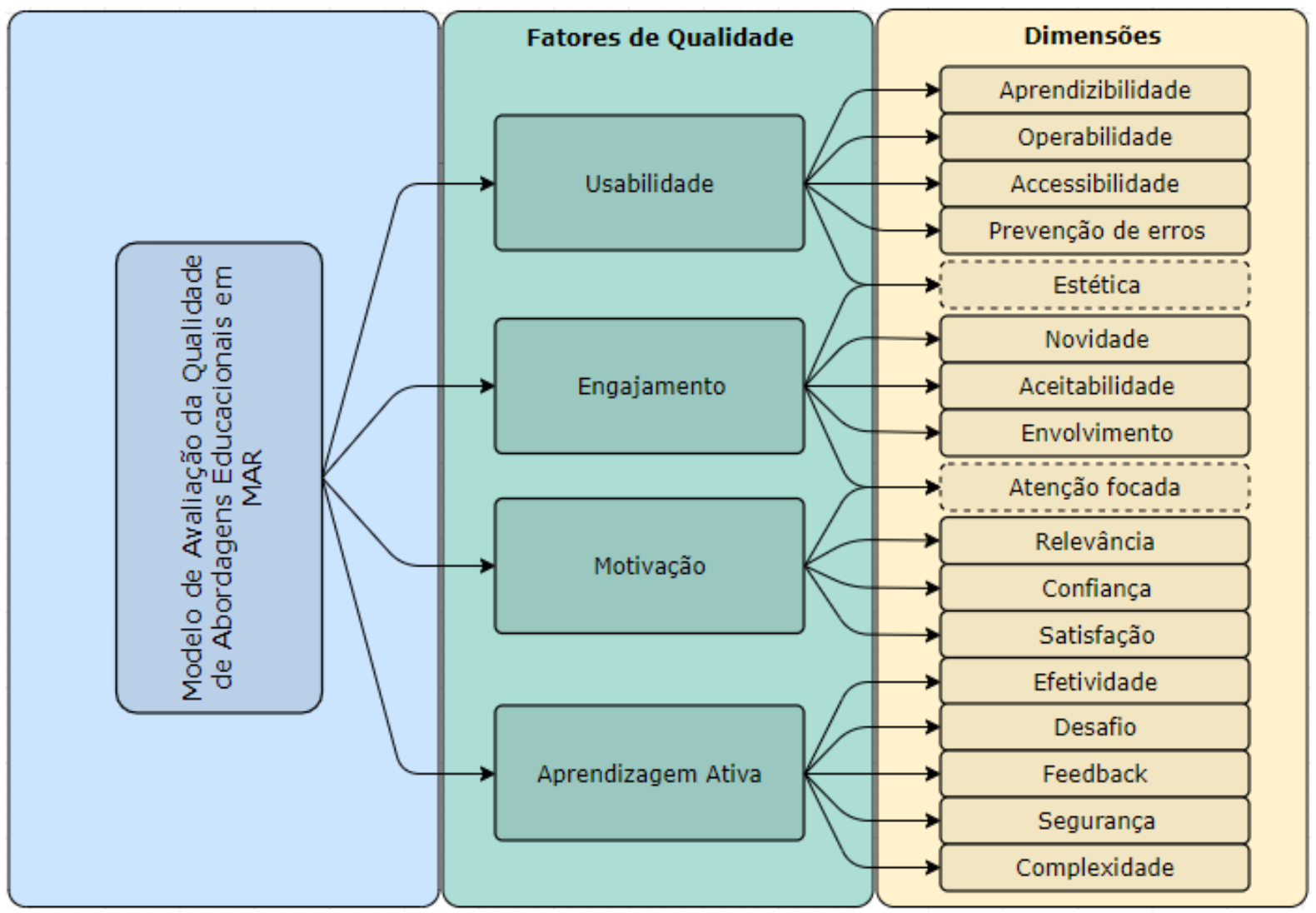

Figura II. Estrutura do Modelo de avaliação MAREEA

Legenda: Derivação dos fatores de qualidade em dimensões de avaliação. As dimensões de avaliação com formatação de borda “-------" pertencem a mais de um fator de qualidade.

Para sistematicamente coletar os dados dos fatores/dimensões, o modelo MAREEA contém um instrumento de medição (questionário) desenvolvido seguindo o método proposto por DeVellis (2016). Tendo como premissa a estrutura do modelo de avaliação, foram consultados questionários padrões para atenderem os fatores de qualidade e suas respectivas dimensões de avaliação. Para atender ao fator de qualidade referente a usabilidade e suas dimensões de avaliação estabelecidas pela ISO/IEC 25010 (2014), foi consultado a pesquisa de Petri, Gresse von Wangenheim \& Borgatto (2018) para a extração e adaptação de 9 itens referentes à usabilidade, os quais foram utilizados na composição do instrumento para a avaliação de abordagens educaciona is em realidade aumentada móvel.

Em relação ao fator de qualidade relacionado ao engajamento foi utilizado como referência a pesquisa de O’Brien \& Cairns (2015) para avaliar esse quesito, que propõem a avaliação de seis dimensões de engajamento (estética, novidade, usabilidade percebida, atenção 
focada, envolvimento e acessibilidade) através de 31 itens. Para elencar os itens que compõem o fator de qualidade de motivação, foi consultada a literatura específica da área que indicou $o$ modelo de Keller (1987), como sendo o mais relevante na atualidade para avaliação de motivação, sendo composto por cinco dimensões (atenção, relevância, confiança e satisfação) e 36 itens que foram incorporados ao instrumento de avaliação de abordagens educaciona is em realidade aumentada móvel.

A respeito do fator de qualidade referente à aprendizagem, foram consultados mais de um questionário e extraídas questões consideradas pertinentes para a avaliação da aprendizagem ativa em abordagens educaciona is em realidade aumentada móvel. Para tanto, foram extraídos setenta e sete itens das pesquisas de Pintrich et al. (1991) e National Research Council (2003).

Após consultar os instrumentos de avaliação específicos para cada fator de qualidade e suas dimensões, foram definidos os itens do questionário que objetivam coletar dados sobre a percepção da qualidade em relação aos recursos de realidade aumentada móvel em contexto educacional. Na primeira versão, 146 itens foram extraídos e classificados para a primeira versão do questionário do modelo MAREEA. Dado o grande número de itens, em um primeiro momento os autores realizaram uma revisão preliminar, com o objetivo de remover possíveis duplicações ou itens fora do contexto analisado. Além disso, também foi determinado o formato de resposta para cada um dos itens do instrumento de medição, definiu-se uma escala de Likert de 5 pontos com alternativas de resposta que variam de discordo totalmente a concordo totalmente.

Após ser definido o objetivo de avaliação, o conjunto de itens e o formato de respostas, outra etapa prevista na metodologia de DeVellis (2016) consiste na revisão dos itens por especialistas. Na próxima seção será apresentada a avaliação inicial do modelo MAREEA com especialistas da área.

\section{AVALIAÇÃO INICIAL DO MODELO MAREEA}

Com o objetivo de avaliar a validade aparente do modelo MARREA foi realizada uma avaliação inicial por meio de um painel de especialistas, etapa prevista nas diretrizes definidas por DeVellis (2016). Para tanto, foram consultados 15 especialistas (11 pesquisadores seniores e 4 pesquisadores juniores) da área de informática e educação, selecionados a partir da identificação de pesquisas envolvendo o uso de tecnologias na educação. A revisão dos especialistas teve como objetivo analisar a clareza, relevância, consistência e completude dos fatores/dimensões do modelo MAREEA e os itens do seu respectivo instrumento de medição, sendo que na oportunidade, os especialistas tinham a possibilidade de remover determinado item ou propor adequações.

Após análise preliminar dos autores, com a finalidade de identificar itens duplicados e fora do contexto desta avaliação, dos 146 itens da primeira versão do instrumento elaborado para o modelo MAREEA, apenas 84 itens foram mantidos e submetidos para a avaliação dos especialistas. Foi elaborado um material digital complementar para contextualizar os especialistas acerca do modelo proposto e como avaliá-la e também foi enviada uma cópia do instrumento de avaliação, para que os especialistas pudessem julgar a validade de cada item incorporado ao instrumento, assim como propor adequações e/ou melhorias na redação dos mesmos. Após a etapa de revisão dos especialistas, foram definidos os fatores de qualidade, dimensões de avaliação e itens de avaliação do instrumento de avaliação do modelo MAREEA, apresentados na Tabela I. 
Tabela I. Instrumento de avaliação do modelo MAREEA

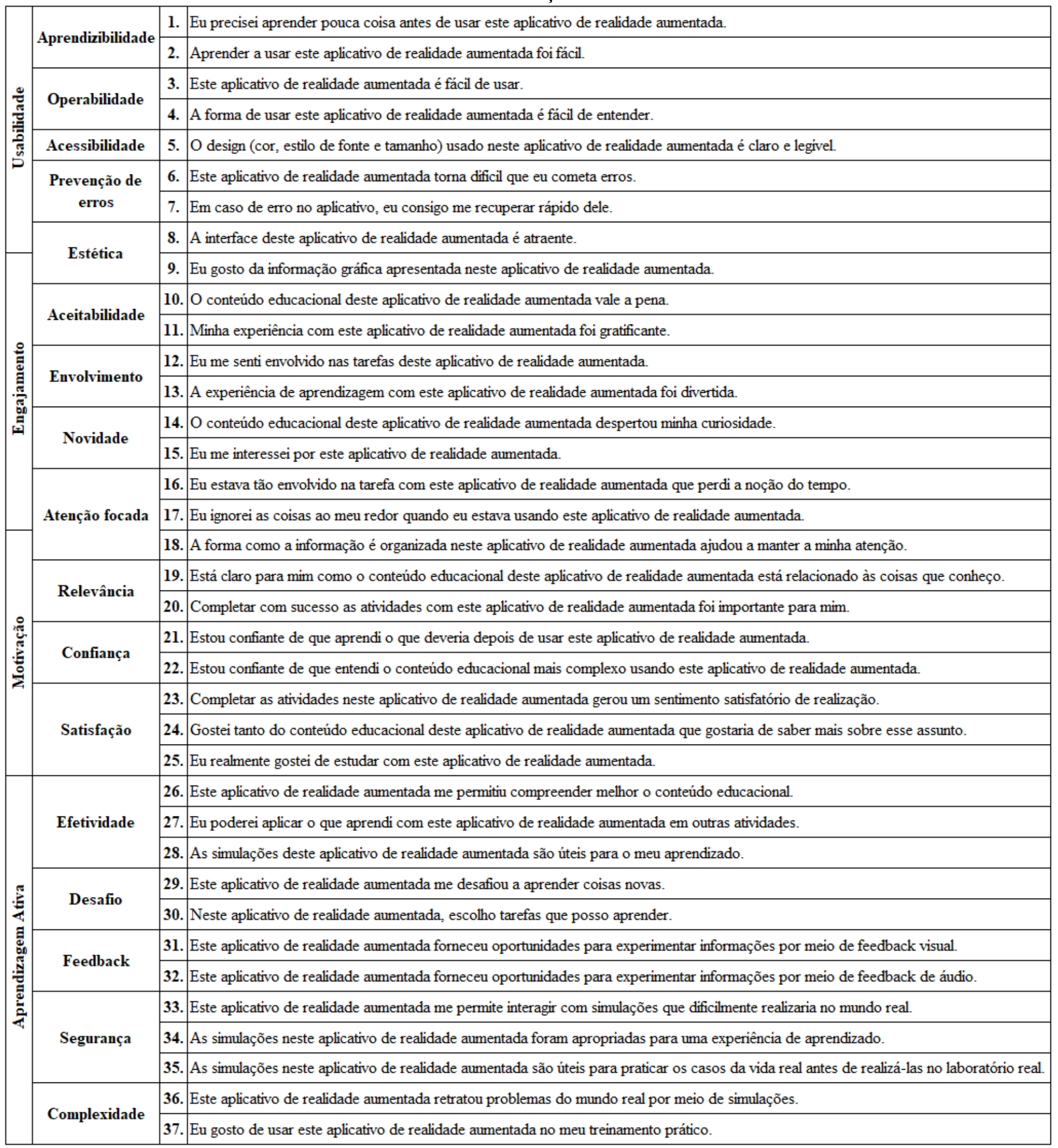

* Para download do modelo de avaliação MAREEA em sua versão Português e/ou Inglês, acesse o link: http://dx.doi.org/10.17632/csb6dbssdp.3. 
Conforme demonstrado na Tabela I, a avaliação da percepção da qualidade de abordagens educacionais em realidade aumentada móvel possui quatro fatores de qualidade, os quais são decompostos em dezoito dimensões de avaliação. Os itens do questionário foram consultados na literatura da área, com vistas a serem capazes de responder às suas respectivas dimensões de avaliação, perfazendo o total de trinta e sete itens para averiguar a qualificação da experiência dos usuários em referência as abordagens educacionais com recursos de realidade aumentada móvel.

De modo geral, os especialistas destacaram a relevância do modelo de avaliação MAREEA para a área de pesquisa, pelo fato de que, atualmente, considera-se uma limitação a falta de recursos para a avaliação destas abordagens educacionais, que fazem o uso de recursos de realidade aumentada em dispositivos móveis. Aspecto também observado no estudo recente de Garzón, Pavón \& Baldiris (2017), no qual evidenciam que os aplicativos de realidade aumentada no contexto educacional são avaliados apenas levando em consideração sua eficácia técnica. Na revisão dos especialistas também foi possível confirmar a completude do modelo MAREEA, indicando que representa fatores importantes para a avaliação da qualidade de abordagens educacionais em realidade aumentada móvel. Entende-se que este resultado foi alcançado em virtude dos fatores de qualidade terem sido extraídos através da revisão sistemática da literatura (Herpich et al., 2019), que analisou diversas pesquisas conduzidas na área e possibilitou uma definição mais precisa dos fatores de qualidade.

Outro destaque dos especialistas refere-se ao rigor científico adotado no desenvolvimento do modelo de avaliação MAREEA, pois a literatura atual descreve que em muitos casos as avaliações são realizadas com questionários desenvolvidos de forma ad-hoc pelos autores, sem uma definição clara do que pretendem medir (Ibáñez \& Delgado-Kloos, 2018). Neste sentido, os especialistas indicaram adequações para os itens de avaliação, de modo a atribuir maior clareza aos mesmos, a fim de facilitar a interpretação dos questionamentos por parte dos futuros avaliadores. Também foram sugeridos pelos especialistas, alterações na redação e na formatação do texto dos itens, assim como houve a recomendação para a exclusão de quarenta e sete itens de avaliação, os quais foram preteridos pelos especialistas em virtude de duplicação e por não atenderem ao contexto que se pretendia avaliar no modelo MAREEA, ficando o instrumento de avaliação ao término desta avaliação com o total de trinta e sete itens. Outro ponto destacado pelos especialistas após a avaliação do modelo MAREEA, envolve a consistência dos fatores de qualidade e a derivação dos seus itens de avaliação, que teve como base a revisão sistemática da literatura e a análise da versão inicial do modelo pelos autores desta pesquisa.

\section{CONSIDERAÇÕES FINAIS}

Neste artigo foi apresentado o desenvolvimento do modelo de avaliação de abordagens educacionais que fazem uso de recursos de realidade aumentada em dispositivos móveis. A partir do objetivo de avaliação foi possível construir o modelo MAREEA, seus fatores de qualidade e dimensões de avaliação, assim como foi possível estabelecer os itens a serem avaliados e o formato das respostas para o instrumento de medição.

Por meio das respostas dos especialistas, foi possível observar que o modelo de avaliação MAREEA atendeu ao objetivo definido para este artigo. Porém, cabe destacar que para comprovar a efetividade do instrumento na avaliação da qualidade de abordagens educacionais em realidade aumentada móvel, se faz necessário completar as etapas previstas na metodologia de construção de escalas de DeVellis (2016), que perpassa para a fase de aplicação dos itens com um grupo de indivíduos, avaliação da validade e confiabilidade dos itens e otimização do tamanho do instrumento. 
A respeito das vantagens oferecidas pelo modelo de avaliação MAREEA, pode-se destacar a aplicabilidade interdisciplinar no âmbito do uso de soluções educacionais com realidade aumentada móvel, dado que o modelo MAREEA pode ser utilizado para avaliar a qualidade de abordagens em diferentes áreas do conhecimento, tais como física, química, informática, entre outras que frequentemente fazem uso de abordagens educacionais com recursos de realidade aumentada móvel. Além disso, como ponto positivo está a redução proporcionada por meio das sugestões dos especialistas, de forma que não haja um excessivo número de fatores de qualidade, dimensões e itens de avaliação, além do tratamento dos termos para permitir maior clareza e acessibilidade durante a sua aplicação por pesquisadores de diferentes áreas de conhecimento para indivíduos de diferentes áreas e faixas etárias.

A fim de validar o modelo MAREEA, esta pesquisa concentrará esforços futuros na condução de estudos de caso para avaliações com indivíduos que utilizam aplicativos com recursos de realidade aumentada móvel no contexto educacional, com vistas a mensurar a sua efetividade no processo de ensino e aprendizagem por meio do uso do modelo MAREEA e também analisar a validade e confiabilidade do modelo MAREEA.

\section{AGRADECIMENTOS}

Esta pesquisa é apoiada pela Coordenação de Aperfeiçoamento de Pessoal de Nível Superior (CAPES) e Conselho Nacional de Desenvolvimento Científico e Tecnológico (CNPq), entidades do governo brasileiro voltadas para o desenvolvimento científico.

\section{REFERÊNCIAS}

Becker, S. A., Cummins, M., Davis, A., Freeman, A., Giesinger, C. H. \& Ananthanarayanan, V. (2017). "NMC Horizon Report: 2017 Higher Education Edition". Austin: Texas.

Borrero, A. M., Sánchez, M. A. M., Márquez, J. M. A. \& Herrera, M. R. S. (2013). “A Complete Solution for Developing Remote Labs". 10th IFAC Symposium Advances in Control Education, pp. 96-101.

Chatzopoulos, D., Bermejo, C., Huang, Z. \& Hui, P. (2017). "Mobile Augmented Reality Survey: From Where We Are to Where We Go". IEEE Access, 5, pp. 6917-6950, 2017.

Chen, P., Liu, X., Cheng, W. \& Huang, R. (2017). "A review of using Augmented Reality in Education from 2011 to 2016". Innovations in Smart Learning, pp. 13-18.

Craig, A. (2013). Understanding augmented reality: Concepts and applications. 296 p.

DeVellis, R. F. (2016). Scale development: Theory and Applications. 4. ed. SAGE Publications.

Frank, J. A. \& Kapila, V. (2017). "Mixed-reality learning environments: Integrating mobile interfaces with laboratory test-beds". Computers \& Education, 110, pp. 88-104.

Garzón, J., Pavón, J. \& Baldiris, S. (2017). Augmented Reality Applications for Education: Five Directions for Future Research. In: Augmented Reality, Virtual Reality, and Compute $\mathbf{r}$ Graphics. AVR 2017. Lecture Notes in Computer Science. De Paolis L., Bourdot P., Mongelli A. (Eds.). Cham: Springer International Publishing, p. 402-414.

Herpich, F., Nunes, F. B., Petri, G., Tarouco, L. M. R. (2019). How Mobile Augmented Reality is applied in Education? A systematic literature review. Creative Education, 10 (7), pp. 1-39. Ibáñez, M. \& Delgado-kloos, C. (2018). "Augmented reality for STEM learning: A systematic review". Computers \& Education, 123, pp. 109-123.

International Standard Organization (ISO). (2014). ISO/IEC 2510: Systems and software engineering - Systems and software Quality Requirements and Evaluation (SQuaRE) System and software quality models. 
Jacques, R., Preece, J. \& Carey, T. (1995). "Engagement as a Design Concept for Multimed ia". Canadian Journal of Educational Communication (CJEC), 24(1), pp. 49-59.

Keller, J. (1987). 'Development and use of the ARCS Model of Instructional Design”. Journal of Instructional Development, 10(2), pp. 2-10.

Maiti, A., Maxwell, A. D. \& Kist, A. A. (2017). 'Using marker based augmented reality and natural user interface for interactive remote experiments". 4th Experiment International Conference, pp. 159-164.

National Research Council. (2003). Evaluating and Improving Undergraduate Teaching in Science, Technology, Engineering, and Mathematics. Washington, DC: The National Academies Press. 232 p.

O'Brien, H. \& Cairns, P. (2015). "An empirical evaluation of the User Engagement Scale (UES) in online news environments". Information Processing and Management, 51(4), pp. 413-427.

Odeh, S., Shanab, S. A. \& Anabtawi, M. (2015). "Augmented Reality Internet Labs versus its Traditional and Virtual Equivalence". International Journal of Emerging Technologies in Learning, 10(3), pp. 4-9.

Petri, G., Gresse von Wangenheim, C. \& Borgatto, A. F. (2018). "MEEGA+, Systematic Model to Evaluate Educational Games". Encyclopedia of Computer Graphics and Games. L. Newton, Ed. Cham: Springer International Publishing, pp. 1-7.

Pintrich, P. R., Smith, D. A. F., Garcia, T. \& Mckeachie, W. J. (1991). A Manual for the Use of the Motivated Strategies for Learning Questionnaire (MSLQ). Technical Report No. 91B-004. National Center for Research to Improve Postsecondary Teaching and Learning: Ann Arbor, MI. $76 \mathrm{p}$.

Preece, J. (2001). "Sociability and usability in online communities: Determining and measuring success". Behavior and Information Technology Journal, 20(5), pp. 347-356.

Prince, M. (2004). "Does active learning work? A review of the research". Journal of Engineering Education, 93(3), pp. 223-232.

Yip, J., Wong, S., Yick, K., Chan, K. \& Wong, K. (2019). "Improving quality of teaching and learning in classes by using augmented reality video". Computers \& Education, 128, pp. 88101. 